\title{
Circular polarization in radio emission from extensive air showers
}

\author{
Olaf Scholten*1,2, T.N.G. Trinh ${ }^{1}$, A. Bonardi ${ }^{3}$, S. Buitink ${ }^{4}$, A. Corstanje ${ }^{3}$, H. Falcke ${ }^{3,5,6}$, \\ B.M. Hare ${ }^{1}$, J.R. Hörandel ${ }^{3,5}$, P. Mitra ${ }^{4}$, K. Mulrey ${ }^{4}$, A. Nelles ${ }^{3,7}$, J.P. Rachen ${ }^{3}$, \\ L. Rossetto ${ }^{3}$, P. Schellart ${ }^{3,9}$, S. ter Veen ${ }^{3,7}$, S. Thoudam ${ }^{3,8}$, T. Winchen $^{4}$ \\ ${ }^{1}$ University of Groningen, KVI Center for Advanced Radiation Technology, 9747 AA Groningen, \\ The Netherlands \\ ${ }^{2}$ Interuniversity Institute for High-Energy, Vrije Universiteit Brussel, Pleinlaan 2, 1050 \\ Brussels, Belgium \\ ${ }^{3}$ Department of Astrophysics/IMAPP, Radboud University, P.O. Box 9010, 6500 GL Nijmegen, \\ The Netherlands \\ ${ }^{4}$ Astrophysical Institute, Vrije Universiteit Brussel, Pleinlaan 2, 1050 Brussels, Belgium \\ ${ }^{5}$ Nikhef, Science Park Amsterdam, 1098 XG Amsterdam, The Netherlands \\ ${ }^{6}$ Netherlands Institute for Radio Astronomy (ASTRON), 7990 AA Dwingeloo, The Netherlands \\ ${ }^{7}$ Department of Physics and Astronomy, University of California, Irvine, CA 92697-4575, USA \\ ${ }^{8}$ Department of Physics and Electrical Engineering, Linnéuniversitetet, 35195 Växjö, Sweden \\ ${ }^{9}$ Department of Astrophysical Sciences, Princeton University, Princeton, NJ 08544, USA \\ E-mail: Scholten@KVI.nl
}

\begin{abstract}
At LOFAR we measure the radio emission from extensive air showers (EAS) in the frequency band of $30-80 \mathrm{MHz}$ in dual-polarized antennas. Through an accurate antenna calibration we can determine the complete set of four Stokes parameters that uniquely determine the linear and circular polarization of the radio signal for an EAS. The observed dependency of the circular polarization on azimuth angle and distance to the shower axis is explained as due to the interfering contributions from the two different radiation mechanisms, a main contribution due to a geomagnetically-induced transverse current and a secondary component due to the Askaryan effect. The measured data show a quantitative agreement with microscopic CORSIKA/CoREAS calculations. Having a very detailed understanding of radio emission from EAS, opens the possibility to use circular polarization as an investigative tool in the analysis of air shower structure, such as for the determination of atmospheric electric fields.
\end{abstract}

35th International Cosmic Ray Conference - ICRC2017

10-20 July, 2017

Bexco, Busan, Korea

*Speaker. 


\section{Introduction}

The understanding of the mechanism of radio emission from extensive air showers (EAS) has reached a level that makes this process suitable for high-precision studies. As a prime example we treat in this contribution the circular polarization of the radiation. As we will show, this is due to a rather subtle interference of the two main emission mechanisms, geomagnetic and charge excess radiation. Because of this high level of understanding of the emission mechanism the radio emission from EAS induced by energetic cosmic rays is a very sensitive way to determine shower properties, such as energy [1,2] and $X_{\max }$, the atmospheric (slant) depth where the number of airshower particles reaches a maximum [3,4]. The latter is a measure of the mass composition of the primary cosmic rays $[5,6]$.

Microscopic models based on first principles have been developed to calculate the radio emission from the EAS such as CoREAS [7] and ZHAireS [8]. Their predictions agree to a high level of precision with measurements at LOFAR (LOw-Frequency ARray) $[9,10]$ and other radio antenna arrays [11], see also Ref. [4].

To get insight in the coherent radiation mechanisms a macroscopic view of the shower is essential. The Lorentz force, $\hat{e}_{\vec{v} \times \vec{B}} \propto \hat{e}_{\vec{v}} \times \hat{e}_{\vec{B}}$, induces a current in the plasma cloud at the shower front that is transverse to the shower direction which is given by $\hat{e}_{\vec{v}} \cdot \hat{e}_{\vec{B}}$ denotes the direction of the geomagnetic field. A secondary contribution [12], due to the net negative charge in the plasma cloud, is also known as Askaryan radiation [13]. The associated current is parallel to $\hat{e}_{\vec{v}}$. In the far-field regime radiation is polarized in the plane spanned by the emitting current and the observer while it is perpendicular to its propagation direction. We thus obtain that the geomagnetic component is polarized in the $\hat{e}_{\vec{v} \times \vec{B}}$ direction while radial for charge-excess radiation. This is also confirmed by observations $[14,15,16,17,18]$.

The radio pulse has also a certain amount of circular polarization [19]. As has recently been shown [20] the circular polarization is caused by a slight, of order $1 \mathrm{~ns}$, time-shift between the pulses emitted by the two emission mechanisms due to a different dependence on the viewing angle to the shower axis $[12,21]$. The circular polarization thus constitutes an accurate test of the differences between the geomagnetic and the charge excess emission mechanisms. At LOFAR we have measured the Stokes parameters [17] for radio emission and shown that these are a convenient way to express the complete polarization of the radio pulse. The data are in excellent agreement with the model calculations.

\section{LOFAR}

LOFAR [22] is a digital radio telescope where for the present measurements we use only the Low Band Antennas (LBAs), each consisting of two inverted V-shaped dipoles where we used the $30-80 \mathrm{MHz}$ band. We usually store $2.1 \mathrm{~ms}$ of data, sampled at $200 \mathrm{Msamples} / \mathrm{s}$ per antenna after a trigger from the LORA scintillator array. Using the reconstructed arrival direction, the polarization directions of the signal are choosen in the direction of the Lorentz force $\hat{e}_{\vec{v} \times \vec{B}}$ and $\hat{e}_{\vec{v} \times \vec{v} \times \vec{B}}$. The instrumental influences induced by the antenna and the amplifier are corrected for in this process. For the present analysis the antenna positions are projected onto the shower plane which is defined as the plane perpendicular to the shower, $\hat{e}_{\vec{v}}$, and going through the core of the shower on the 
ground. A detailed description of the offline analysis of the data for cosmic ray science can be found in $[9,3,17]$.

The Stokes parameters are most easily expressed in terms of the complex voltages $\mathscr{E}_{i}=E_{i}+i \hat{E}_{i}$,

$$
\begin{aligned}
I & =\frac{1}{n} \sum_{0}^{n-1}\left(|\mathscr{E}|_{i, \vec{v} \times \vec{B}}^{2}+|\mathscr{E}|_{i, \vec{v} \times \vec{v} \times \vec{B}}^{2}\right) \\
Q & =\frac{1}{n} \sum_{0}^{n-1}\left(|\mathscr{E}|_{i, \vec{v} \times \vec{B}}^{2}-|\mathscr{E}|_{i, \vec{v} \times \vec{v} \times \vec{B}}^{2}\right) \\
U+i V & =\frac{2}{n} \sum_{0}^{n-1}\left(\mathscr{E}_{i, \vec{v} \times \vec{B}} \mathscr{E}_{i, \vec{v} \times \vec{v} \times \vec{B}}^{*}\right),
\end{aligned}
$$

Here $E_{i}$ is sample $i$ of the electric field component in either the $\hat{e}_{\vec{v} \times \vec{B}}$ or the $\hat{e}_{\vec{v} \times \vec{v} \times \vec{B}}$ polarization direction and $\hat{E}_{i}$ its Hilbert transform [17]. For the present analysis the summations are performed over $n=5$ samples, of $5 \mathrm{~ns}$ each, centered around the pulse maximum. The linear-polarization direction makes an angle $\psi=\frac{1}{2} \tan ^{-1}(U / Q)$ with the $\vec{v} \times \vec{B}$-axis. The circular polarization is given by $V / I$. It is convenient to define $W^{2}=I^{2}-\left(Q^{2}+U^{2}+V^{2}\right)$ which is a measure of the difference in structure of the signal in the two polarization directions, likely due to noise, see Ref. [20] for a more extensive discussion.

\section{Interpretation}

Circular polarization can be understood as the rotation of the polarization direction over the duration of the pulse, due to a time lag between the charge excess and geomagnetic pulses. To quantify this it is conceptually easiest to consider a signal of fixed frequency $\omega$. The radiallypolarized charge-excess pulse,

$$
\mathscr{E}_{C}(t)=E_{C} e^{i \omega(t-\Delta t)}\left(\cos \phi \hat{e}_{\vec{v} \times \vec{B}}+\sin \phi \hat{e}_{\vec{v} \times \vec{v} \times \vec{B}}\right),
$$

thus has a time lag of $\Delta t$ with respect to the transverse-current pulse,

$$
\mathscr{E}_{T}(t)=E_{T} e^{i \omega t} \hat{e}_{\vec{v} \times \vec{B}}
$$

The angular position of the antenna with respect to the $\hat{e}_{\vec{v} \times \vec{B}}$-direction is denoted by $\phi$. The Stokes parameters now read

$$
\begin{aligned}
I & =E_{T}^{2}+E_{C}^{2}+2 E_{T} E_{C} \cos \phi \cos \omega \Delta t \\
Q & =E_{T}^{2}+E_{C}^{2} \cos (2 \phi)+2 E_{T} E_{C} \cos \phi \cos \omega \Delta t \\
U & =E_{C}^{2} \sin (2 \phi)+2 E_{T} E_{C} \sin \phi \cos \omega \Delta t \\
V & =2 E_{T} E_{C} \sin \phi \sin \omega \Delta t .
\end{aligned}
$$

Extreme values for the circular polarization are reached for $\phi=\pi / 2$ and $\phi=-\pi / 2$. Thus $V / U=$ $\tan \omega \Delta t$ measures the the time-lag, between charge excess and geomagnetic radiation.

As an illustrative example we focus on an air shower for which the radio signal was detected in six LOFAR stations. The Stokes parameters extracted for each LOFAR antenna is shown versus 


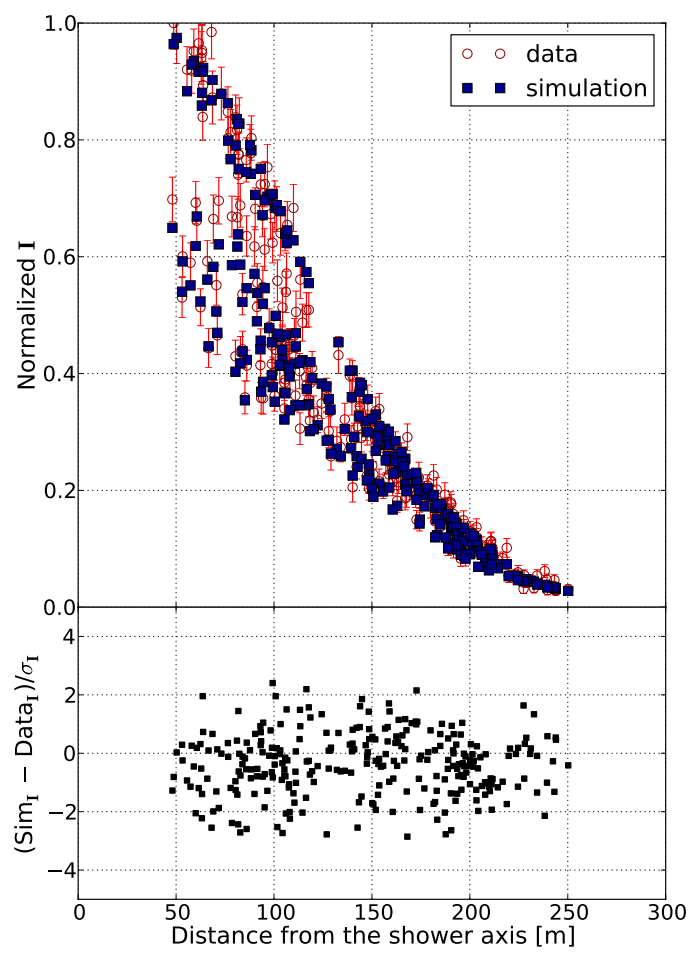

Figure 1: The measured intensity footprint of a shower (open red circles) is compared to the results of a CoREAS simulation (filled blue squares). The standard deviation is denoted by $\sigma$.
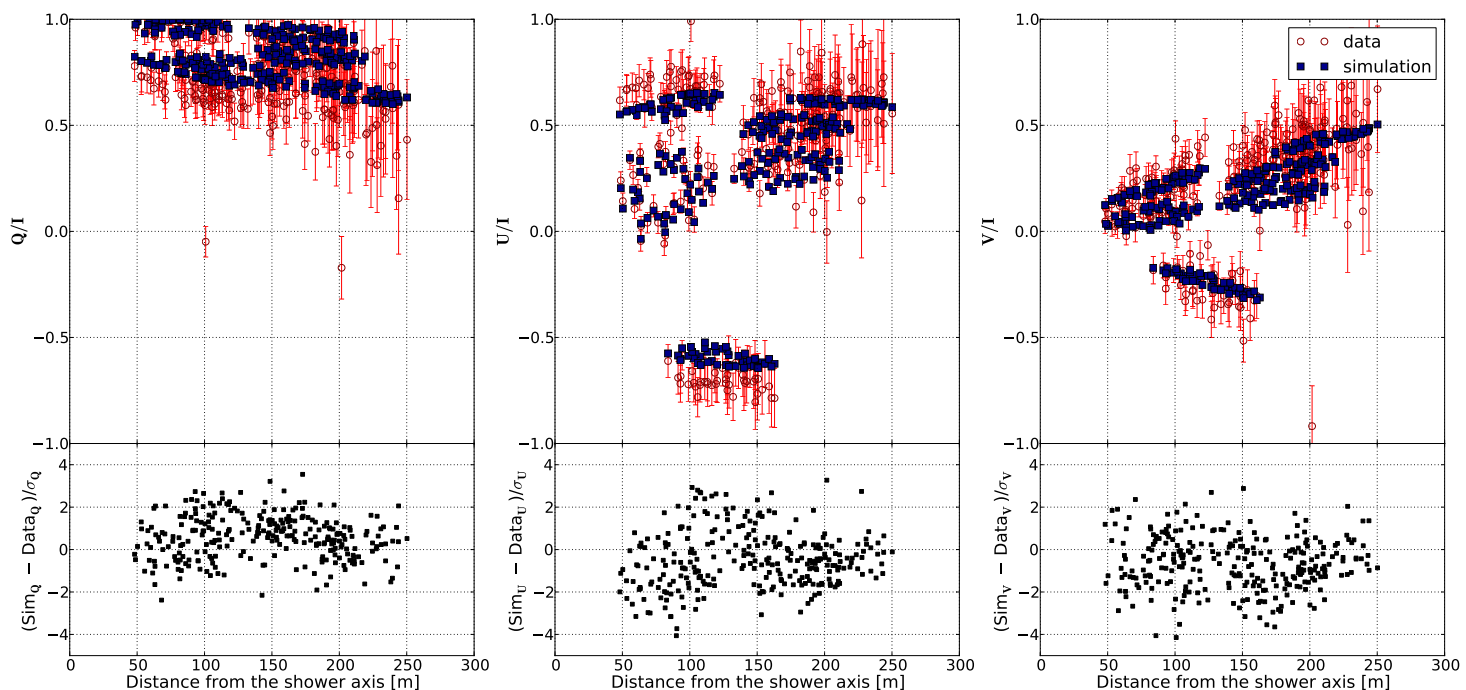

Figure 2: The normalized Stokes parameters for the air shower shown in Fig.1 where the meaning of the different symbols is also indicated. 
distance to the shower axis in Fig. 1 and Fig. 2. The CoREAS simulation was done for $X_{\max }=$ $659 \mathrm{~g} / \mathrm{cm}^{2}$, shower energy $E=6.24 \times 10^{17} \mathrm{eV}$, and zenith angle $\theta=26^{\circ}$. The calculated values for $W / I$ (not shown) are vanishingly small while the measured values for $W / I$, due to noise, increase from less than a percent at small distances to about than $10 \%$ at larger distances. The scatter of the points at a fixed distance is due to the dependence of the Stokes parameters on the azimuthal position of the antenna, see Eq. (3.1).

Understanding the difference in the relative timing of the two radio pulse requires looking in detail into the two emission mechanisms. Taking the $z$-axis along the shower and the $x$-axis along $\hat{e}_{\vec{v} \times \vec{B}}$ the vector potential due to transverse current is

$$
A^{\mu}=j^{\mu} / D=j^{x}\left(t_{\text {ret }}\right) / D
$$

where $j^{\mu}$ is the four current induced in the plasma cloud of the EAS and $D$ the retarded distance [12]. The retarded time is related to the emission height, $t_{\mathrm{ret}}=-z / c$. The current induced by the Lorentz force is roughly proportional the the number of charged particles in the plasma cloud, $N\left(t_{\text {ret }}\right)$, thus $j^{x}\left(t_{\text {ret }}\right) \propto N\left(t_{\text {ret }}\right)$ [23]. The charge excess in the plasma cloud, that moves with the speed of light to Earth, gives rise to a time and $z$-component of the current. Since the charge excess, like the geomagnetic current, is also roughly proportional to the lepton number in the cloud we obtain $j^{0}\left(t_{\text {ret }}\right) \propto N\left(t_{\text {ret }}\right)$. Only due to a different dependence on air pressure and shower age a slight difference in the height (or retarded time) dependence of the two currents is induced and the transverse current reaches its maximum at somewhat larger heights than the charge excess [12].

To understand the circular polarization one has to go through the derivation of the radiation field from the vector potential

$$
\vec{E}=-\vec{\nabla} A^{0}-d \vec{A} / d t .
$$

This yields $\vec{E}=-d \vec{A} / d t$ for the transverse current contribution. Since this is like the emission from an varying electric current we call this magnetic emission. For the charge excess contribution we obtain $\vec{E}=-d A^{0} / d r$. Since this is similar to the radiation from a charge we call this electric and it is polarized in the radial, $\hat{r}$ direction. For a fixed emission height the time and radial derivatives are related through [23] $d t_{\text {ret }} / d t \approx c(R / r) d t_{\text {ret }} / d r$ where $R$ is the distance to the emission point. Even if the charge excess and geomagnetic currents are assumed to have the same dependence on height in the atmosphere we now have obtained that the main contribution to the received intensity is lower in the atmosphere for charge excess than for geomagnetic. Numerical simulations show that this difference is about $1 \mathrm{~km}$, depending on zenith angle of the cosmic ray.

This difference in emission height is schematically indicated in Fig. 3 left side where the curves labeled 'GeoMagnetic' and 'ChargeX' denote the height dependence of contribution of the different processes to the received signal. Due to the resulting difference in arrival time of the peak intensity for the two components the net polarization direction of the detected signal will the rotate over an angle $\phi$. The angle $\phi$ is the azimuthal position of the observer with respect to the $\vec{v} \times \vec{B}$-axis and is thus the same as the angle between the polarization directions of the geomagnetic and the chargeexcess emissions, as indicated in the figure. It is thus clear that the circular polarization depends on the azimuthal position of the observer, as can be read from Eq. (3.1). This azimuthal dependence of the circular polarization can also be seen from the figure at the right side of Fig. 3 where the data and simulation for the footprint of the Stokes parameter $V$ are compared. As expected, $\hat{e}_{\vec{v} \times \vec{B}}$ 
is the axis of anti-symmetry, where the circular polarization has a different sign for points above or below this axis.
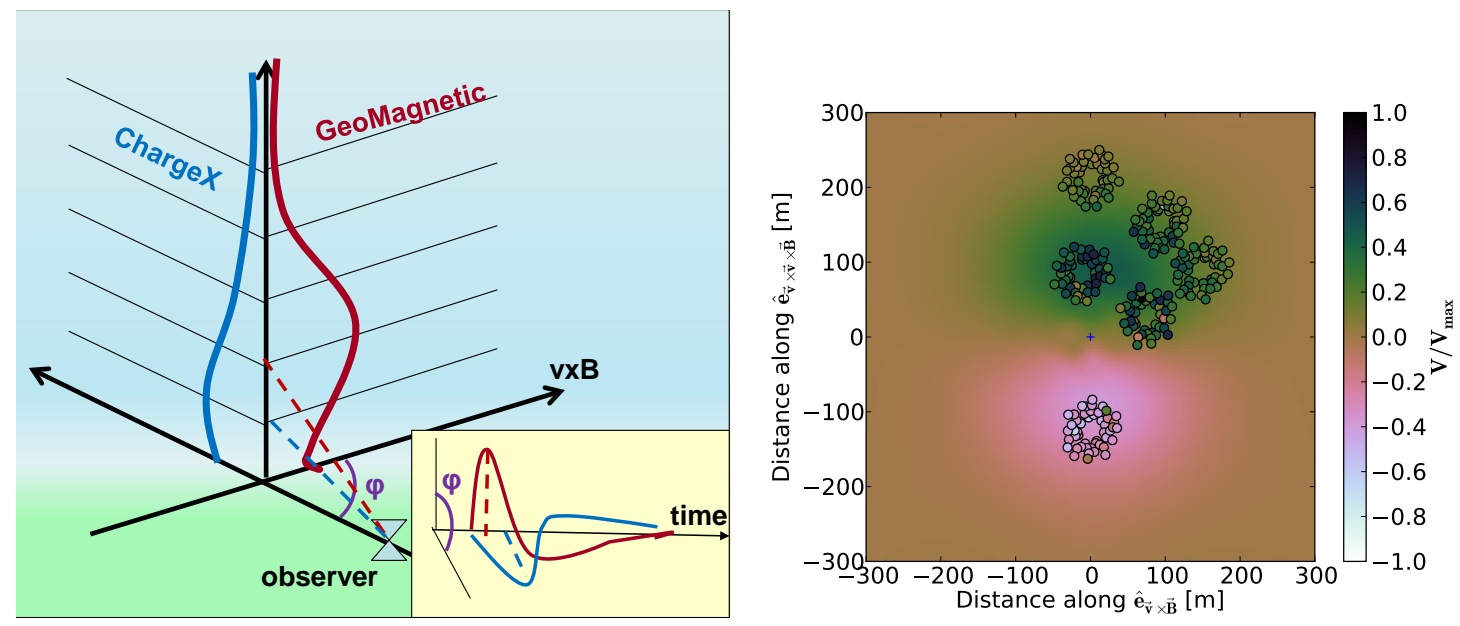

Figure 3: Schematic picture showing the source of circular polarization. Right side shows The footprint of the value of the Stokes $V$-parameter for a measured air shower. The background color shows the results of the CoREAS simulation while the coloring in the small circles presents the data. This is the same data as shown in Fig.2 (right most panel).

An equivalent explanation, based on the arguments given in the introduction is that the polarization is given by the projection of the inducing current on a direction perpendicular to the propagation direction of the radiation. The amplitude is proportional to the magnitude of the projected current. For geomagnetic emission the inducing current is in the $\vec{v} \times \vec{B}$ direction which is already almost perpendicular to the line of sight. For charge excess radiation the inducing current is along the shower axis which implies that the projection perpendicular to the line of sight is small near the core of the shower and increases with distance. This also implies that emission from lower parts of the EAS are favored due to the projection factor. This leads to the same conclusion as we just arrived at, i.e. that even with the same height dependence of the inducing currents the charge excess radiation is effectively emitted at lower heights compared to geomagnetic radiation resulting in circular polarization of the signal.

\section{Conclusions \& Summary}

We have made a detailed investigation of the circular polarization of the radio emission of an EAS recorded under fair weather circumstances. We have given an interpretation based on macroscopic currents as are expected in an EAS and shown that it is due to a slight time delay between the charge excess and the geomagnetic pulse. This interpretation is supported by microscopic calculations using CoREAS that shows an almost perfect agreement with the data measured at LOFAR for all Stokes parameters.

Having shown that the full set of measured Stokes parameters for an event detected under fair weather conditions are quantitatively and quantitatively understood allows to use EAS as a diagnostics tool to extract atmospheric electric fields [17, 24]. 


\section{Acknowledgements}

The LOFAR cosmic ray key science project acknowledges funding from an Advanced Grant of the European Research Council (FP/2007-2013) / ERC Grant Agreement n. 227610. The project has also received funding from the European Research Council (ERC) under the European Union's Horizon 2020 research and innovation programme (grant agreement No 640130). We furthermore acknowledge financial support from FOM, (FOM-project 12PR3041-3) and NWO (Top Grant 614001-454, and Spinoza Prize SPI 78-409). AN is supported by the DFG (research fellowship NE 2031/1-1).

LOFAR, the Low Frequency Array designed and constructed by ASTRON, has facilities in several countries, that are owned by various parties (each with their own funding sources), and that are collectively operated by the International LOFAR Telescope foundation under a joint scientific policy.

\section{References}

[1] A. Nelles et al., "The radio emission pattern of air showers as measured with LOFAR - a tool for the reconstruction of the energy and the shower maximum", J. Cosm. and Astrop. Phys. 05, 018 (2015); arXiv:1411.7868.

[2] A. Aab et al., "Energy Estimation of Cosmic Rays with the Engineering Radio Array of the Pierre Auger Observatory”, Phys. Rev. D 93, 122005 (2016); arXiv:1508.04267.

[3] S. Buitink et al., "Method for high precision reconstruction of air shower $X_{\max }$ using two-dimensional radio intensity profiles", Phys. Rev. D 90, 082003 (2014).

[4] T. Huege, "Radio detection of cosmic ray air showers in the digital era", Phys. Rep. 620, 1 (2016); arXiv:1601.07426.

[5] W.D. Apel et al., "Reconstruction of the energy and depth of maximum of cosmic-ray air-showers from LOPES radio measurements”, Phys. Rev. D 90, 062001 (2014).

[6] S. Buitink et al., "A large light-mass component of cosmic rays at $10^{17} U^{\prime 1} 10^{17.5}$ electronvolts from radio observations", Nature 531, 70 (2016).

[7] T. Huege, M. Ludwig, and C. James, "Simulating radio emission from air showers with CoREAS", AIP Conf. Proc. 1535, 128 (2012).

[8] J. Alvarez-Muñiz et al., "Monte Carlo simulations of radio pulses in atmospheric showers using ZHAireS”, Astropart. Phys. 35, 325 (2012).

[9] P. Schellart et al., "Detecting cosmic rays with the LOFAR radio telescope", Astron. \& Astrophys. 560, A98 (2013); arXiv:1311.1399.

[10] A. Nelles et al., "Measuring a Cherenkov ring in the radio emission from air showers at 110-230 MHz with LOFAR", Astropart. Phys. 65, 11 (2015); arXiv:1411.6865.

[11] D. Ardouin et al., "Radiodetection of cosmic ray extensive air showers: Upgrade of the CODALEMA experiment”, Int. J. Mod. Phys. A 20, 6869 (2005).

[12] K.D. de Vries et al., "The lateral distribution function of coherent radio emission from extensive air showers: Determining the chemical composition of cosmic rays", Astropart. Phys. 34, 267 (2010). 
[13] G.A. Askaryan, "Excess negative charge of an electron-photon shower and its coherent radio emission”, Sov. Phys. JETP 14, 441 (1962).

[14] V. Marin, for the CODALEMA Collaboration, "Charge excess signature in the CODALEMA data; Interpretation with SELFAS2", Proc. 32 ${ }^{\text {nd }}$ ICRC, Beijing, 1, 291 (2011).

[15] H. Schoorlemmer, for the Pierre Auger Collaboration, "Results from polarization studies of radio signals induced by cosmic rays at the Pierre Auger Observatory", Nucl. Instr. and Meth. A 662, S134 (2012).

[16] A. Aab et al., "Probing the radio emission from air showers with polarization measurements.", Phys. Rev. D 89, 052002 (2014); arXiv:1402.3677.

[17] P. Schellart et al., "Polarized radio emission from extensive air showers measured with LOFAR", J. Cosm. and Astrop. Phys. 1410, 014 (2014); arXiv:1406.1355.

[18] A. Bellétoile et al., "Evidence for the charge-excess contribution in air shower radio emission observed by the CODALEMA experiment", Astropart. Phys. 69, 50 (2015)

[19] H. Schoorlemmer, "Tuning in on cosmic rays.", PhD thesis, Radboud Univeristy Nijmegen, ISBN: 978-90-9027039-5, 2008.

[20] O. Scholten et al., "Measurement of the circular polarization in radio emission from extensive air showers confirms emission mechanisms", Phys. Rev. D 94, 103010 (2016).

[21] K. D. de Vries, O. Scholten, and K. Werner, "The air shower maximum probed by Cherenkov effects from radio emission”, Astropart. Phys. 45, 23 (2013); arXiv:arXiv:1304.1321.

[22] M. P. van Haarlem et al., "LOFAR: The LOw-Frequency ARray”, Astron. \& Astrophys. 556, A2 (2013); arXiv:1305.3550.

[23] O. Scholten, K. Werner, and F. Rusydi, “A Macroscopic Description of Coherent Geo-Magnetic Radiation from Cosmic Ray Air Showers", Astropart. Phys. 29, 94 (2008); K. Werner and O. Scholten, "Macroscopic Treatment of Radio Emission from Cosmic Ray Air Showers based on Shower Simulations", Astropart. Phys. 29, 393-411 (2008).

[24] T.N.G. Trinh et al., "Thunderstorm electric fields probed by extensive air showers through their polarized radio emission", Phys. Rev. D 95, 083004 (2017). 